\title{
Patient education for hyperphosphatemia management: Improving outcomes while decreasing costs?
}

\author{
Mirey Karavetian ${ }^{1}$, Rana Rizk ${ }^{2,3}$ \\ 'Department of Health Sciences, College of Natural and Health Sciences, Zayed University, Dubai, United Arab Emirates \\ 2Institut National de Santé Publique, d'Épidémiologie Clinique et de Toxicologie (INSPECT-LB), Faculty of Public Health, \\ The Lebanese University, Fanar, Lebanon \\ ${ }^{3}$ Department of Health Services Research, Care and Public Health Research Institute (CAPHRI), Maastricht University, \\ Maastricht, The Netherlands
}

Of the countless lifestyle adaptations required by hemodialysis patients, behavioral changes associated with the management of hyperphosphatemia, such as the adoption of a low phosphate diet and judicious intake of phosphate binders, are probably the most complicated and challenging $[1,2]$. Non-adherence to low phosphate diets, which are likely the most common dietary restrictions required from this patient population, ranges between 19 and $57 \%[3,4]$. Likewise, non-adherence to phosphate binders is common, ranging between 22 and $74 \%$, with more than half of hemodialysis patients not following their prescribed regimens [5]. In line with this background, it is unsurprising that more than a decade after the first international clinical practice guidelines for chronic kidney disease-mineral bone disorder were issued [6], a wide gap persists between recommended and measured serum phosphorus levels among hemodialysis patients in clinical practice. Hyperphosphatemia is the most common mineral abnormality among this patient population, with nearly one in two hemodialysis patients being hyperphosphatemic [3]. While the pharmaceutical industry has responded by developing new agents, little

Received January 29, 2018; Revised January 31, 2018;

Accepted January 31, 2018

Correspondence: Rana Rizk

Department of Health Services Research, Care and Public Health Research Institute (CAPHRI), Maastricht University, 6200 MD Maastricht, The Netherlands.E-mail:r.rizk@maastrichtuniversity.nl

ORCID: http://orcid.org/oooo-0002-8850-6502

Copyright (C 2018 by The Korean Society of Nephrology

(c) This is an open-access article distributed under the terms of the Creative Commons Attribution Non-Commercial License (http://creativecommons.org/licenses/by nc-nd/4.0/), which permits unrestricted non-commercial use, distribution, and reproduction in any medium, provided the original work is properly cited. importance has been given to patient education programs, specifically counseling to foster behavioral changes, in clinical practice, despite mounting high-quality evidence regarding the effectiveness of this intervention [7].

In this issue of Kidney Research and Clinical Practice, Lim et al [8] assessed the impacts of nutrition and pharmacologic education on serum phosphate, calciumphosphate product, and adherence to a low phosphate diet, i.e., dietary phosphate intake and phosphorus-toprotein ratio, self-reported compliance with phosphate binder prescriptions, prescribed bioequivalent dose of binders and knowledge regarding proper intake timing, as well as nutritional status, among other parameters. After random assignment to experimental groups, 48 patients received one 30-minute individualized educational session with a dietitian and another session with a pharmacist, while the control group received conventional care without education sessions. The investigators explored changes over the short (one-month post-education) and long-term (two or three months post-education). The authors reported no significant changes in any of the study parameters between the intervention and the control groups, either over the short- or long-term. The absence of any effect associated with patient education intervention could be partly explained by the study limitations acknowledged by the authors, specifically, the baseline parameters of the sample. More than two-thirds of the study sample had good phosphate control, and mean baseline serum phosphate levels that were within the recommended range according to international renal guidelines [6]; moreover, the mean baseline compli- 
ance score was medium. These figures are suggestive of good adherence to treatment among the patients in the study sample, which is unlike the situation commonly observed among hemodialysis patients described in the international literature. The lack of elucidation regarding essential features to design a successful patient education program for optimal hyperphosphatemia management may also explain the absence of any effect associated with the intervention. Specifically, the most valuable features may include sustainability of at least monthly education, for over 6 months, as well as a focus on counseling for behavioral change, rather than solely focusing on the transfer of knowledge [9].

It is becoming incumbent upon the healthcare profes- sional community to acknowledge that education programs are indispensable for the optimal management of hemodialysis patients in routine care. It is also essential to recognize that in conjunction with these programs, refining patient knowledge alone does not lead to actual behavioral changes and improvements in outcomes, specifically for hyperphosphatemia management. Indeed, there is evidence that hemodialysis patients with better knowledge actually have worse phosphate control [4], because reasons for non-adherence to the treatment of hyperphosphatemia in end-stage renal disease extend beyond poor knowledge. Important factors include patient demographic, clinical, personality and psychosocial characteristics. such as the beliefs about the necessity of

Table 1. Features of a comprehensive and effective patient education program to manage hyperphosphatemia in hemodialysis patients (adapted from references [2] and [9])

\begin{tabular}{|c|c|}
\hline Component & Features \\
\hline \multirow[t]{4}{*}{ Human } & Led by trained nurses, dietitians or other ancillary healthcare providers \\
\hline & Multidisciplinary team approach where depression is also treated \\
\hline & Patients involved in preparation of the intervention \\
\hline & Patients' families and friends involved in education initiatives \\
\hline \multirow[t]{10}{*}{ Information } & Hyperphosphatemia, its complications, and means of control \\
\hline & Relevant blood tests, and review of blood tests' results with the patient \\
\hline & Dietary: \\
\hline & - Appropriate food choices \\
\hline & - Phosphorus-to-protein ratio of foods \\
\hline & - Avoidance of phosphate additives \\
\hline & - Training to prepare suitable meals \\
\hline & Phosphate binder: \\
\hline & - Mode of action \\
\hline & - Role and benefit in lowering serum phosphate \\
\hline \multirow{3}{*}{$\begin{array}{l}\text { Counseling theory } \\
\text { and strategy }\end{array}$} & Use of counseling theories, such as the trans theoretical model of behavioral change \\
\hline & Use of counseling strategies, such as motivational interviewing, self-monitoring, and self-regulation \\
\hline & Initiatives for patient empowerment, such as the Phosphate Education Program \\
\hline \multirow[t]{2}{*}{ Format } & Individualized (one-on-one) or group education \\
\hline & Tailored to the patient's lifestyle, environment, career, ethnicity, cultural background, and socioeconomic status \\
\hline \multirow[t]{3}{*}{ Tools } & $\begin{array}{l}\text { Handouts and visual tools: colorful informational booklets; lists of common phosphate-rich and -poor foods; } \\
\text { food alternatives; posters }\end{array}$ \\
\hline & Games and puzzles \\
\hline & Recipes adapted to taste and culture \\
\hline Timing & Before the hemodialysis session \\
\hline Duration & At least 6 months \\
\hline Frequency & At least monthly \\
\hline \multirow[t]{4}{*}{ Binder properties } & Reduce pill size and burden \\
\hline & Improve palatability \\
\hline & Reduce associated adverse effects \\
\hline & Introduce electronic monitoring devices (to help patients to remember to take their medication and support adherence) \\
\hline
\end{tabular}


and concerns about the potential adverse consequences of phosphate binders, as well as food fatigue, or gradually becoming disinterested and subsequently less adherent to the low phosphate diet, in addition to perceived social support from friends, family and renal staff $[1,5]$.

When educating hemodialysis patients on serum phosphate control, educators should shift from traditional knowledge-based, expert-centered guidance, to patientcentered counseling approaches that focus on empowerment and shared decision-making, with an ultimate goal of fostering behavioral change and preventing relapse [9].

\section{Effective patient education for hyperphosphatemia management: how can we achieve that?}

"One of the greatest opportunities to improve patient outcomes will probably come not from discovering new treatments, but from more effective delivery of existing therapies" [10]. Patient education programs are uniquely potent in this regard. Undeniably, whenever trialed or meta-analyzed, patient education has proven to be an effective adjunct to other means of hyperphosphatemia management [7]. It is now well established that frequent, long-term, individualized education, delivered by trained healthcare professionals using cognitive and/or behavioral strategies and providing patients with problemsolving skills and ongoing reinforcement, is an effective approach for managing hyperphosphatemia among hemodialysis patients without compromising their nutritional status [2,9]. Table 1 delineates essential features of an effective patient education program for controlling serum phosphate. Education for hyperphosphatemia management should be sustained in all patients, including those who are compliant, in order to ensure they maintain healthy behaviors and subsequently normal serum phosphate levels [1].

\section{Patient education: an economically attractive alternative?}

Given continually rising healthcare costs and limited budgets, cost considerations are gaining more and more attention in decisions about adoption of health technologies. There is little doubt that patient education is a promising and economically attractive intervention in hyperphosphatemia management. Emerging evidence

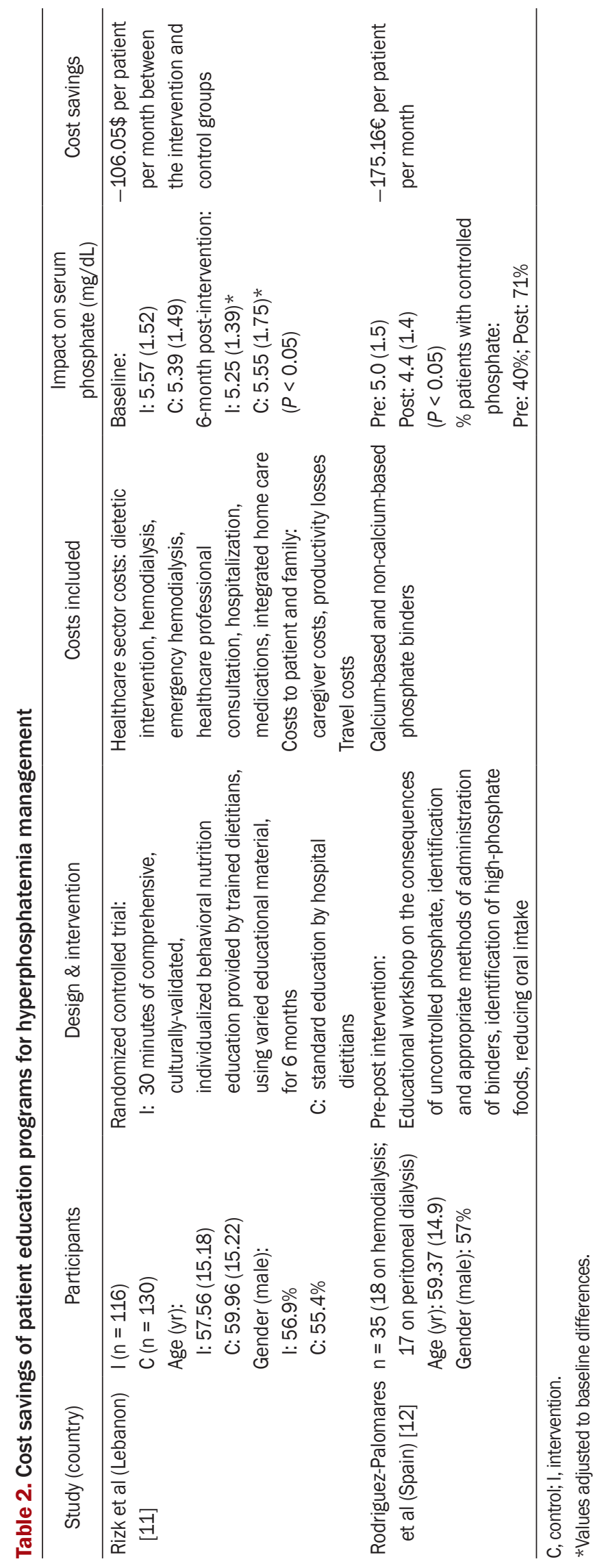


clearly indicates that patient education has low direct costs and is an inexpensive intervention within hemodialysis care [9]. The implementation of this intervention could generate tremendous cost savings and significant clinical improvement $[11,12]$. Rizk et al [11] found that the cost savings generated by the implementation of an intensive nutrition education for hyperphosphatemia management in hemodialysis units offsets the cost of the education program more than eight times. Table 2 provides examples of the monumental cost-saving impacts of two patient education programs targeting low phosphate diet and phosphate binder intake, respectively. Accordingly, it seems reasonable to plan a powered randomized controlled trial, extended with a model-based analysis, that is specifically designed to investigate the cost-effectiveness of patient education programs for hyperphosphatemia management in hemodialysis patients. The provision of solid evidence about the economic efficiency of this intervention could encourage health policymakers to implement similar programs in routine clinical practice. This may in turn contribute to more efficient allocation of public health budgets, while simultaneously enhancing quality of care and patient outcomes.

In conclusion, patient-tailored counseling and sufficient time spent with patients are associated with improvements in serum phosphate control, as long as constant reinforcement measures are in place. Patient education programs could also provide valuable opportunities for containing costs, which are much-needed in modern nephrology care.

\section{Conflicts of interest}

All authors have no conflicts of interest to declare.

\section{References}

[1] Kalantar-Zadeh K. Patient education for phosphorus management in chronic kidney disease. Patient Prefer Adherence 7:379-390, 2013

[2] Covic A, Rastogi A. Hyperphosphatemia in patients with ESRD: assessing the current evidence linking outcomes with treatment adherence. BMC Nephrol 14:153, 2013
[3] Denhaerynck K, Manhaeve D, Dobbels F, Garzoni D, Nolte C, De Geest S. Prevalence and consequences of nonadherence to hemodialysis regimens. Am J Crit Care 16:222-235, 2007

[4] Durose CL, Holdsworth M, Watson V, Przygrodzka F. Knowledge of dietary restrictions and the medical consequences of noncompliance by patients on hemodialysis are not predictive of dietary compliance. J Am Diet Assoc 104:35-41, 2004

[5] Karamanidou C, Clatworthy J, Weinman J, Horne R. A systematic review of the prevalence and determinants of nonadherence to phosphate binding medication in patients with end-stage renal disease. BMC Nephrol 9:2, 2008

[6] National Kidney Foundation. K/DOQI clinical practice guidelines for bone metabolism and disease in chronic kidney disease. Am J Kidney Dis 42(4 Suppl 3):S1-S201, 2003

[7] Milazi M, Bonner A, Douglas C. Effectiveness of educational or behavioral interventions on adherence to phosphate control in adults receiving hemodialysis: a systematic review. JBI Database System Rev Implement Rep 15:9711010, 2017

[8] Lim E, Hyun S, Lee JM, et al. Effects of education on lowphosphate diet and phosphate binder intake to control serum phosphate among maintenance hemodialysis patients: A randomized controlled trial. Kidney Res Clin Pract 37:69-76, 2018

[9] Karavetian M, de Vries N, Rizk R, Elzein H. Dietary educational interventions for management of hyperphosphatemia in hemodialysis patients: a systematic review and meta-analysis. Nutr Rev 72:471-482, 2014

[10] Pronovost PJ, Nolan T, Zeger S, Miller M, Rubin H. How can clinicians measure safety and quality in acute care? Lancet 363:1061-1067, 2004

[11] Rizk R, Hiligsmann M, Karavetian M, Evers SMAA. Cost-effectiveness of dedicated dietitians for hyperphosphatemia management among hemodialysis patients in Lebanon: results from the Nutrition Education for Management of Osteodystrophy trial. J Med Econ 20:1024-1038, 2017

[12] Rodriguez-Palomares JR, Cancino MCJ, Blazquez Collado LB, et al. Educating your patient is cost-effective: It reduces serum phosphate and saves D 200 per patient per month. Nefrologia 37:103-105, 2017 